\title{
Hydrogen Abstraction by Chlorine Atom from Amino Acids: Remarkable Influence of Polar Effects on Regioselectivity
}

\author{
Robert J. O’Reilly, ${ }^{\dagger}$ Bun Chan, ${ }^{\dagger}$ Mark S. Taylor, ${ }^{\dagger}$ Sandra Ivanic, ${ }^{\dagger}$ George B. Bacskay, ${ }^{\dagger}$ Christopher J. Easton, ${ }^{+,}$ \\ and Leo Radom ${ }^{*+}$
}

${ }^{+}$ARC Centre of Excellence for Free Radical Chemistry and Biotechnology and School of Chemistry, University of Sydney, Sydney,
NSW 2006, Australia

${ }^{\ddagger}$ Research School of Chemistry, Australian National University, Canberra, ACT 0200, Australia

Supporting Information

ABSTRACT: Quantum chemistry computations have been used to investigate hydrogen-atom abstraction by chlorine atom from protonated and $\mathrm{N}$-acetylated amino acids. The results are consistent with the decreased reactivity at the backbone $\alpha$ carbon and adjacent side-chain positions that is observed experimentally. The individual effects of $\mathrm{NH}_{3}{ }^{+}, \mathrm{COOH}$, and NHAc substituents have been examined and reveal important insights. The $\mathrm{NH}_{3}{ }^{+}$group in isolation is found to be deactivating at the $\alpha$-position, while the acetamido group is activating. For the COOH group, polar effects lead to a contrathermody-

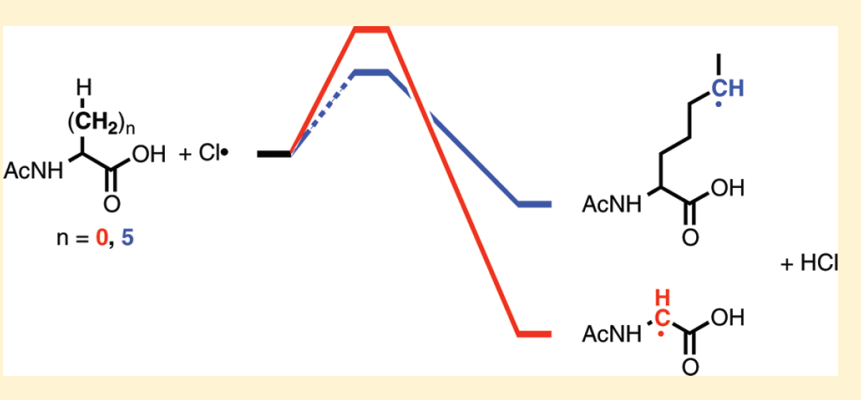
namic deactivation of the thermodynamically most favorable $\alpha$-abstraction. In the $N$-acetylamino acid, the $\alpha$-position is deactivated by the combined inductive effect of the substituents and the presence of an early transition structure, again overriding the greater thermodynamic stability of the $\alpha$-centered radical product. Deactivation of the $\alpha$-, $\beta$-, and $\gamma$-positions results in a peculiar stability for amino acids and peptides and their derivatives with respect to radical degradation.

\section{INTRODUCTION}

Hydrogen-atom abstraction from the $\alpha$-position of protonated amino acids and their $N$-acylated derivatives (i.e., the structural motifs representing the fundamental building blocks of peptides and proteins) is a critical event which, when coupled with subsequent reactions of the resultant $\alpha$-centered radicals, culminates in fragmentation of peptides and proteins. ${ }^{1}$ The ensuing fragmentation processes have been identified as important steps in the development of a wide range of pathological conditions such as aging, cancer, and neurodegenerative disorders. ${ }^{2}$ From a biological perspective, it is therefore of great relevance that, although $\mathrm{C}_{\alpha}-\mathrm{H}$ abstractions do occur to some extent, a plethora of experimental studies reported in the past five decades have revealed that the $\mathrm{C}_{\alpha}-\mathrm{H}$ (and to a lesser extent the $\mathrm{C}_{\beta}-\mathrm{H}$ ) bonds of such structures are in fact relatively inert toward abstraction by electrophilic radicals such as $\mathrm{OH}^{\bullet}$ and $\mathrm{Cl}^{\bullet}{ }^{3}$ In the present investigation, we use quantum chemistry computations to examine this behavior.

Early experimental studies of the regioselectivity of the chlorination reactions of amino acids demonstrated that, under strongly acidic conditions, chlorination failed to occur at the $\alpha$ position but occurred instead at distal positions along the alkyl chains. ${ }^{4}$ It was also demonstrated that the $\alpha$-positions of $N$-acylated amino acids are resistant to hydrogen-atom abstraction by $\mathrm{Cl}^{\bullet}$. For example, chlorination of $N$-benzoylvaline methyl ester was found to result exclusively in the $\beta$ and $\gamma$ products, with no product derived from the $\alpha$-position. ${ }^{5}$ In addition, electron paramagnetic resonance studies of the chlorination reactions of $\mathrm{N}$-acetylvaline showed no evidence for the formation of an $\alpha$-centered radical, with signals observed only for the $\beta$ - and $\gamma$-centered radicals. ${ }^{6}$ The results for the $N$-acylated amino acids are particularly important because their $\alpha$-carbon-centered radicals are subject to particularly large stabilizing effects, associated with captodative interactions, ${ }^{7}$ and therefore the relatively slow abstraction rates to produce them represents a substantial contrathermodynamic effect.

The relatively slow hydrogen-abstraction rates, despite in some cases resulting in the formation of highly stabilized radical products, may be rationalized in terms of two related effects. First, the $\alpha$-carbon is electron deficient due to the $\sigma$-electronwithdrawing character of the substituents $\left(\mathrm{NH}_{3}{ }^{+}, \mathrm{COOH}\right.$, and NHAc). For electrophilic radicals such as $\mathrm{Cl}^{\circ}$, the interaction between the incipient polar $\mathrm{Cl}-\mathrm{H}$ and the electron-deficient radical center results in a destabilization of the transition structure. ${ }^{3,8-10}$ Such effects are often referred to in the literature as "polar effects". ${ }^{11}$ Second, such transition structures are often "early", with little degree of $\mathrm{C}-\mathrm{H}$ bond cleavage, and thus they do not benefit significantly from the (captodative, in the case of the $N$-acylated amino acids) stabilization effects in the radical products. This picture is supported by the observation that the corresponding hydrogen-atom abstractions by $\mathrm{Br}^{\bullet}$ (which is

Received: June 27, 2011

Published: September 26, 2011 
Table 1. Experimentally Observed Relative Rates ${ }^{a}$ for Abstraction of Methylene Hydrogen Atoms from Protonated and $N$-Acetyl Amino Acids by Chlorine ${ }^{b}$

$\begin{array}{ccc}\text { position } & \mathrm{RCH}\left(\mathrm{NH}_{3}{ }^{+}\right) \mathrm{CO}_{2} \mathrm{H} & \mathrm{RCH}(\mathrm{NHAc}) \mathrm{CO}_{2} \mathrm{H} \\ \alpha & \leq 0.05 & \leq 0.20 \\ \beta & 0.05 & 0.35 \\ \gamma & 0.91 & 2.7 \\ \delta & 3.7 & 5.0\end{array}$

${ }^{a}$ From ref 13 . Reaction rates relative to that for the methylene hydrogens of phenylalanine. ${ }^{b} \mathrm{R}=\mathrm{H}$ for $\alpha$, and $\mathrm{R}=$ alkyl for $\beta, \gamma$, and $\delta$.

substantially less electrophilic than $\mathrm{Cl}^{\bullet}$ ) do favor abstraction from the $\alpha$-position, because the "late" transition structures do benefit from the radical stabilization effects. ${ }^{12}$

Taking into account all of these previous findings, Watts and Easton recently reported a comprehensive study of radical chlorination reactions for a significantly more diverse range of protonated and $\mathrm{N}$-acetylated amino acid derivatives, as well as small peptides, than had been investigated previously. ${ }^{13}$ Consistent with previous reports, their results again demonstrated that the $\alpha$-positions are essentially unreactive, with chlorination proceeding with greater facility as one moves down the chain away from the functional groups. The experimental findings were also taken to suggest that these so-called "polar effects" are long-range and propagate well beyond the $\alpha$-position (Table 1).

From a biological perspective, the apparent lack of reactivity toward hydrogen-atom abstraction (by electrophilic radicals such as $\mathrm{Cl}^{\bullet}$ and $\mathrm{OH}^{\bullet}$ ) near the backbone of amino acids and peptides led Watts and Easton to suggest this as one reason why nature may have selected $\alpha$-amino acids as the building blocks of peptides and proteins. ${ }^{13}$ In the absence of such effects, it would be reasonable to expect that these biopolymers would rapidly degrade in an environment where they are constantly surrounded by and processing radicals. ${ }^{14,15}$

Despite the longstanding experimental interest in the regioselectivity of hydrogen-atom abstractions of amino acids and their derivatives by $\mathrm{Cl}^{\circ}$, we are not aware of any quantum chemical investigations that have appeared in the literature examining this effect. There are some distinct advantages compared with experimental studies in investigating these reactions using a quantum chemical approach. Perhaps the most important is that any ambiguity that might arise from different protonation states of the substrates or from competing reaction mechanisms is avoided. For example, the recently reported experimental radical chlorinations ${ }^{13}$ were performed in trifluoroacetic acid, where protonation of the NHAc and $\mathrm{COOH}$ substituents and involvement of $\mathrm{N}$-chloroamines and amides as reaction intermediates may complicate the interpretation of the results. By contrast, the calculations involve only hydrogen-atom transfer from substrates specifically substituted with $\mathrm{NHAc}, \mathrm{COOH}$, and $\mathrm{NH}_{3}{ }^{+}$groups. We have therefore used computational quantum chemistry in the present study to try to provide a better understanding of the lack of reactivity of the amino acid backbone and adjacent side-chain positions in the chlorination reactions. ${ }^{16}$

We have examined hydrogen-atom abstractions by chlorine from the $\alpha$-methylene moieties of both protonated and $N$ acetylated glycine, and the $\beta-\varepsilon$-methylenes of $\alpha$-aminoheptanoic acid, and have determined associated thermodynamic parameters. In addition, we have considered the effect of each of the respective functional groups $\mathrm{NHAc}, \mathrm{COOH}$, and $\mathrm{NH}_{3}{ }^{+}$in isolation. To this

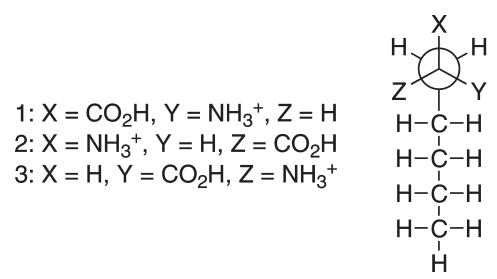

Figure 1. Possible conformations of protonated $\alpha$-aminoheptanoic acid.

end, we note that experimental kinetic data are available in the literature for the chlorination of carboxylic acids, ${ }^{17}$ but only qualitative trends have been reported for protonated amines, ${ }^{18}$ and it appears that $\mathrm{N}$-alkylacetamides have not been investigated in this context.

\section{COMPUTATIONAL DETAILS}

In this section, we outline the main features of our computational approach, while full details are provided in the Supporting Information. Gas-phase computations were performed using Gaussian $03^{19}$ and Gaussian $09,{ }^{20}$ with geometries of all structures optimized at the BHandH-LYP/ $6-31+G(d, p)$ level of theory. ${ }^{21}$ Calculations were carried out for a variety of conformations. As an illustrative example, for protonated $\alpha$-aminoheptanoic acid we have examined abstraction of each nonequivalent methylene hydrogen atom in each of three conformations (Figure 1), leading to potentially 24 nonequivalent transition structures and 24 nonequivalent product radicals (see also section S1 of the Supporting Information).

Initially we focused our attention on the calculation of enthalpic barriers $\left(\Delta H_{\mathrm{c}}^{\ddagger}\right)$ using a conventional approach. Thus, we carried out single-point energy calculations on the BHandH-LYP/6-31+G(d,p) geometries, using the higher-level B2K-PLYP double-hybrid density functional theory procedure, ${ }^{22}$ in conjunction with the $\mathrm{A}^{\prime} \mathrm{VTZ}$ basis set (where $\mathrm{A}^{\prime} \mathrm{VTZ}$ denotes a basis set consisting of cc-pVTZ for $\mathrm{H}$, aug-ccpVTZ for first-row elements, and aug-cc-pV(T+d)Z for $\mathrm{Cl}) .^{23,24} \mathrm{To}$ obtain the ZPVE and $H_{\text {vib }}$ corrections necessary to calculate the reaction enthalpies and enthalpic barriers at $298 \mathrm{~K}$, we used BHandH-LYP/6-31 $+\mathrm{G}(\mathrm{d}, \mathrm{p})$ harmonic vibrational frequencies scaled using appropriate literature scale factors. ${ }^{25}$ Likewise, the $S_{\text {vib values that are required for }}$ the determination of free energies in subsequent variational transition state theory (VTST) calculations were obtained from appropriately scaled BHandH-LYP/6-31+G(d,p) frequencies.

Variational effects may be of importance when considering hydrogenatom abstraction reactions, especially when the enthalpic barriers are small or negative. This applies to many of the barriers in the present investigation, and so we have also calculated variational free energy barriers $\left(\Delta G_{\mathrm{v}}^{\ddagger}\right)$ using Polyrate $2010 \mathrm{~A}^{26}$ in conjunction with Gaussian $09 .^{20}$ The Polyrate portions of our VTST calculations were performed at the BHandH-LYP $/ 6-31+G(d, p)$ level. We then refined the location of the variational maxima on the underlying free energy surface at the $\mathrm{B} 2 \mathrm{~K}$ PLYP level using a mixed basis set designated 6-31+G(3df,2d,2p), comprising $6-31+\mathrm{G}(2 \mathrm{~d}, 2 \mathrm{p})$ for first-row elements and hydrogen, and $6-31+\mathrm{G}-$ $(3 \mathrm{df}, 2 \mathrm{p})$ for chlorine. ${ }^{27}$ Our final reported variational barriers were obtained through performing single-point B2K-PLYP/A'VTZ energy calculations at the B2K-PLYP/6-31+G(3df,2d,2p) variational transition structures.

Solvation corrections at each point on the reaction path were obtained at the M05-2X/6-31G(d) level using the SMD-IEFPCM model ${ }^{28}$ for acetic acid $(\mathrm{AcOH}),{ }^{29}$ allowing a modified variational TS in solution to be obtained. We have briefly examined the effect of quantum mechanical tunneling ${ }^{26}$ in the case of abstraction from the $\alpha$-position of protonated glycine, which has the largest barrier among the reactions examined and therefore can be expected to be the most susceptible to tunneling effects. The transmission coefficients at $298 \mathrm{~K}$, obtained with the zero-curvature 
Table 2. Energies of Reactant Complexes Relative to the Species in Isolation $\left(298 \mathrm{~K}, \mathrm{~kJ} \mathrm{~mol}^{-1}\right)$

\begin{tabular}{cccc}
\hline \multicolumn{1}{c}{ structure } & $\Delta H_{\text {gas }}$ & $\Delta G_{\text {gas }}$ & $\Delta G_{\mathrm{AcOH}}$ \\
\hline$\left[\right.$ protonated glycine $\left.\cdots \mathrm{Cl}^{\bullet}\right]$ & -15.7 & +9.2 & +27.5 \\
{$\left[N\right.$-acetylglycine $\left.\cdots \mathrm{Cl}^{\bullet}\right]$} & -22.2 & +8.8 & +4.8 \\
\hline
\end{tabular}

tunneling ${ }^{30}$ and small-curvature tunneling ${ }^{31}$ methods, are 3.1 and 7.6, respectively. As the predicted rate enhancements are less than an order of magnitude, and we are concerned largely with relative rates (that will benefit from some cancellation of errors), we have not deemed it necessary to include tunneling effects more generally.

For chlorine atom, we have included a correction to entropy $\left(298 \mathrm{~K},-1.73 \mathrm{~kJ} \mathrm{~mol}^{-1}\right)$ associated with the contribution of the low-lying ${ }^{2} \mathrm{P}_{3 / 2}$ and ${ }^{2} \mathrm{P}_{1 / 2}$ electronic states to the electronic partition function, as well as a spin-orbit correction $\left(3.52 \mathrm{~kJ} \mathrm{~mol}^{-1}\right)$. We have assumed that the spin-orbit correction associated with $\mathrm{Cl}^{\circ}$ in the transition structures is fully quenched. Finally, reaction free energies and free energy barriers for the abstraction of hydrogen at the $\alpha$-position in the protonated and $\mathrm{N}$-acetylated amino acids have been obtained from glycine derivatives. This approach was used in order to be consistent with what was done experimentally. ${ }^{13}$

\section{RESULTS AND DISCUSSION}

3.1. Importance of Reactant Complexes. Bimolecular reactions may involve complexes along the reaction path, which, if lower in energy than the isolated species, may be important as reference points for the calculation of reaction barriers. In fact, a recent report ${ }^{32}$ attributed the unfavorability of abstraction of hydrogen from the $\alpha$-position of amino acids by $\mathrm{OH}^{\bullet}$ precisely to the formation of a particularly stable complex, which was suggested to present a kinetic trap for abstraction from this position. While the complex of interest in that study involves interactions (between $\mathrm{OH}^{\bullet}$ and the amido groups within the substrate) that do not exist in the systems examined in the present study, ${ }^{32}$ we nonetheless felt it important to consider whether the formation of reactant complexes might be important in the situations that we are examining, i.e., in determining the barriers for hydrogen-atom abstractions by $\mathrm{Cl}^{\bullet}$ from protonated amino acids and N-acetylated amino acids, given that both sets of systems show resistance to radical attack at the $\alpha$-position. To address this issue, we have located complexes associated with the interaction of $\mathrm{Cl}^{\bullet}$ with protonated glycine and $\mathrm{N}$-acetylglycine and calculated their energies (both on the enthalpic and free energy surfaces) relative to the sum of the energies of the separated species (Table 2).

Considering first the case of protonated glycine, it is evident that, while the reactant complex is stabilized with respect to the isolated reactants on the enthalpic surface $\left(\Delta H_{\text {gas }}=-15.7\right.$ $\left.\mathrm{kJ} \mathrm{mol}{ }^{-1}\right)$, such a complex is destabilized when one takes into account the unfavorable entropic contributions $\left(\Delta G_{\text {gas }}=+9.2\right.$ $\left.\mathrm{kJ} \mathrm{mol}^{-1}\right)$. Thus, our calculations suggest that, in the gas phase, this complex lies $9.2 \mathrm{~kJ} \mathrm{~mol}^{-1}$ higher in free energy than the isolated species. This energy separation is further increased upon moving to the solution phase $\left(\Delta G_{\mathrm{AcOH}}=+27.5 \mathrm{~kJ} \mathrm{~mol}^{-1}\right)$. Analogously, for $\mathrm{N}$-acetylglycine, a complex also exists with an enthalpy lower than that of the isolated species (by $22.2 \mathrm{~kJ} \mathrm{~mol}^{-1}$ ). However, as in the case of protonated glycine, the gas-phase relative free energy of such a complex is positive $\left(+8.8 \mathrm{~kJ} \mathrm{~mol}^{-1}\right)$, and this again is the case upon inclusion of solvent corrections $\left(+4.8 \mathrm{~kJ} \mathrm{~mol}^{-1}\right.$ in $\left.\mathrm{AcOH}\right)$. Our calculations thus suggest that it is unlikely that reactant complexes play a significant role in determining the kinetics of hydrogen-atom abstractions by $\mathrm{Cl}^{\bullet}$ in amino acid derivatives of this type. ${ }^{33}$

3.2. General Features for Hydrogen-Atom Abstractions from Amino Acids and Related Species. In the following sections, we will consider hydrogen-atom abstractions by $\mathrm{Cl}^{\circ}$ from the protonated and $N$-acetylated forms of glycine and $\alpha$ aminoheptanoic acid. Furthermore, to examine the individual contributions of the three substituents, we also consider the effect of each of the $\mathrm{COOH}, \mathrm{NH}_{3}{ }^{+}$, and NHAc substituents in isolation. Results of these investigations are provided in Tables 3 and 4 . Table 3 contains quantities calculated in the gas phase at $298 \mathrm{~K}$, including enthalpies of reaction $(\Delta H)$, free energies of reaction $(\Delta G)$, conventional enthalpic barriers $\left(\Delta H_{\mathrm{c}}^{*}\right)$ (i.e., barriers obtained by single-point energy calculations on the classical BHandH-LYP/6-31+G(d,p) transition structures $)$, conventional free energy barriers $\left(\Delta G_{\mathrm{c}}^{*}\right)$, and enthalpic $\left(\Delta H_{\mathrm{v}}^{*}\right)$ and entropic $\left(-\mathrm{T} \Delta S_{\mathrm{v}}^{\mp}\right)$ contributions to the overall variational gas-phase free energy barriers $\left(\Delta G_{\mathrm{v}}{ }^{\ddagger}\right)$. Table 4 presents condensed-phase free energies of reaction $\left(\Delta G_{\text {soln }}\right)$, variational free energy barriers $\left(\Delta G_{\text {gas }}{ }^{\ddagger}=\Delta G_{\mathrm{v}}{ }^{\ddagger}\right)$ in the gas phase, and contributions from changes in gas-phase free energies (arising from solvent-induced changes in the variational transition structure) $\left(\Delta \Delta G_{\text {gas } / / \text { solv }}{ }^{\ddagger}\right)$ and solvation free energies $\left(\Delta \Delta G_{\text {solv }}{ }^{\ddagger}\right)$ to the overall solution-phase variational free energy barriers $\left(\Delta G_{\text {soln }}{ }^{\ddagger}\right)$. The relationship between these quantities is $\Delta G_{\text {soln }}{ }^{\ddagger}=\Delta G_{\text {gas }}{ }^{\ddagger}+\Delta \Delta G_{\text {gas } / / \text { solv }}{ }^{\neq}$ $+\Delta \Delta G_{\text {solv }}^{\stackrel{*}{*} \text {. In the light of our finding that reactant complexes }}$ are unlikely to play an important role in determining the kinetics of these reactions, all energies in Tables 3 and 4 are calculated relative to separated reactants.

We first note from Table 3 that, in general, there is a reasonable correlation between the trends in the reaction enthalpies $(\Delta H)$ and the barriers $\left(\Delta H_{\mathrm{c}}^{\ddagger}\right.$ and $\left.\Delta H_{\mathrm{v}}^{\ddagger}\right)$, which is in accord with the Bell-Evans-Polanyi principle. Exceptions are found for $n$-heptanoic acid and $N$-acetylglycine, where the barriers for hydrogen abstraction are largest (or least negative) at the $\alpha$-position, despite this leading to the most exothermic reaction. We find that, in general, both the conventional and variational enthalpic barriers are in accord with the observed experimental trends for the protonated and $N$-acetylamino acids, such that the barriers decrease from the $\alpha$-position to the $\varepsilon$-position. However, in many cases, the enthalpic barriers are negative, particularly for those obtained with the conventional approach. When higherlevel B2K-PLYP potential energy surfaces are used (via an IRCmax-type ${ }^{34}$ procedure) and entropic effects are included, the vast majority of the variational free energy barriers $\left(\Delta G_{\mathrm{v}}{ }^{\ddagger}\right)$ are positive and reproduce the experimentally observed trends for the protonated and $\mathrm{N}$-acetyl amino acids and for heptanoic acid.

In general, we find that the variational free energy barriers $\left(\Delta G_{\mathrm{v}}^{\stackrel{f}{f}}\right)$ can differ significantly from those obtained with a conventional approach $\left(\Delta G_{c}{ }^{\ddagger}\right)$, with the largest difference $(21.3 \mathrm{~kJ}$ $\mathrm{mol}^{-1}$ ) associated with abstraction from the $\alpha$-position of $N$ hexylacetamide (Table 3). Thus, with the exception of the abstraction reactions from the $\alpha$-carbons of either the protonated amino acid or the protonated amine, abstractions from the $\alpha$ positions of other substrates are subject to substantial variational effects, culminating in the variational free energy barriers being significantly larger than the conventional barriers. These results highlight the importance of using high-level potential energy surfaces and considering variational effects when studying lowbarrier hydrogen-atom abstractions by $\mathrm{Cl}^{\circ}$. Nonetheless, while the conventional barriers can be quantitatively very different from the corresponding variational barriers, we again emphasize 
Table 3. Gas-Phase Reaction Enthalpies $(\Delta H)$, Reaction Free Energies $(\Delta G)$, Conventional Enthalpic and Free Energy Barriers $\left(\Delta H_{c_{+}}^{\ddagger}\right.$ and $\left.\Delta G_{c}^{\ddagger}\right)$, and Enthalpic $\left(\Delta H_{v}^{\ddagger}\right)$ and Entropic $\left(-T \Delta S_{v}^{\ddagger}\right)$ Contributions to the Overall Variational Free Energy Barriers $\left(\Delta G_{\mathrm{v}}^{\mp}\right)\left(\mathrm{kJ} \mathrm{mol}^{-1}, 298 \mathrm{~K}\right)$

\begin{tabular}{|c|c|c|c|c|c|c|c|c|}
\hline \multicolumn{2}{|l|}{ Species } & $\Delta H$ & $\Delta G$ & $\Delta H_{\mathrm{c}}^{\ddagger}$ & $\Delta G_{\mathrm{c}}^{\ddagger}$ & $\Delta H_{\mathrm{v}}^{\ddagger}$ & $-\mathrm{T} \Delta S_{\mathrm{v}}^{\ddagger}$ & $\Delta G_{v}^{\ddagger}$ \\
\hline & $\alpha^{\mathrm{a}}$ & 0.4 & -3.4 & 45.2 & 80.3 & 45.6 & 35.1 & 80.7 \\
\hline $\mathrm{OH}$ & $\beta$ & -10.3 & -24.3 & -10.4 & 25.8 & -2.8 & 33.2 & 30.4 \\
\hline $\mathrm{NH}_{3}{ }^{+}$ & $\gamma$ & -23.7 & -32.3 & -40.1 & -2.1 & -20.8 & 33.2 & 12.4 \\
\hline & $\delta$ & -12.8 & -26.3 & -41.0 & -9.1 & -36.9 & 43.2 & 6.3 \\
\hline & $\varepsilon$ & -15.1 & -26.8 & -56.9 & -14.6 & -48.9 & 45.3 & -3.6 \\
\hline & $\alpha$ & 8.8 & -2.6 & 31.8 & 60.8 & 32.9 & 29.6 & 62.5 \\
\hline $\mathrm{NH}_{3}{ }^{+}$ & $\beta$ & -12.8 & -25.6 & -13.2 & 19.9 & -7.3 & 33.8 & 26.4 \\
\hline & $\gamma$ & -22.8 & -32.2 & -40.9 & -5.7 & -19.1 & 34.3 & 15.2 \\
\hline & $\delta$ & -12.1 & -24.9 & -43.0 & -7.2 & -35.9 & 40.7 & 4.8 \\
\hline & $\varepsilon$ & -14.8 & -26.6 & -51.1 & -12.0 & -46.0 & 43.9 & -2.1 \\
\hline & $\alpha$ & -34.6 & -43.3 & -5.7 & 27.5 & 3.7 & 33.4 & 37.1 \\
\hline & $\beta$ & -12.9 & -26.1 & -17.7 & 15.4 & -3.9 & 30.7 & 26.8 \\
\hline & $\gamma$ & -14.2 & -26.5 & -25.6 & 7.0 & -7.7 & 30.2 & 22.5 \\
\hline & $\delta$ & -13.9 & -26.5 & -24.2 & 8.1 & -6.4 & 29.2 & 22.9 \\
\hline & $\varepsilon$ & -15.2 & -27.6 & -25.6 & 6.0 & -6.9 & 29.1 & 22.2 \\
\hline & $\alpha$ & -15.3 & -29.4 & -26.2 & 3.3 & -7.3 & 27.4 & 20.2 \\
\hline & $\beta$ & -14.3 & -28.5 & -27.1 & 3.3 & -8.8 & 28.8 & 20.0 \\
\hline & $\gamma$ & -14.4 & -28.7 & -27.3 & 3.1 & -9.0 & 28.9 & 19.9 \\
\hline & $\delta$ & -14.3 & -28.5 & -27.1 & 3.3 & -8.8 & 28.8 & 20.0 \\
\hline & $\varepsilon$ & -15.3 & -29.4 & -26.2 & 3.3 & -7.3 & 27.4 & 20.2 \\
\hline & $\alpha^{\mathrm{a}}$ & -77.0 & -82.5 & -13.8 & 20.5 & -0.6 & 30.2 & 29.6 \\
\hline & $\beta$ & -9.0 & -24.6 & -15.7 & 15.0 & -4.0 & 30.4 & 26.4 \\
\hline $\mathrm{NHAC}$ & $\gamma$ & -14.7 & -26.0 & -22.4 & 11.9 & -6.2 & 29.7 & 23.5 \\
\hline & $\delta$ & -13.4 & -26.4 & -25.4 & 6.1 & -6.8 & 29.7 & 22.9 \\
\hline & $\varepsilon$ & -15.1 & -27.5 & -25.9 & 6.1 & -6.5 & 29.8 & 23.3 \\
\hline & $\alpha^{\mathrm{b}}$ & -37.3 & -45.2 & -41.1 & -6.5 & -22.4 & 37.2 & 14.8 \\
\hline $\mathrm{NH}$ & $\beta$ & -11.5 & -23.5 & -28.3 & 2.8 & -12.2 & 31.8 & 19.6 \\
\hline & $\gamma$ & -14.0 & -26.5 & -28.8 & 4.6 & -12.0 & 33.0 & 21.0 \\
\hline & $\delta$ & -13.8 & -26.1 & -27.8 & 4.9 & -11.0 & 32.0 & 21.0 \\
\hline & $\varepsilon$ & -15.2 & -27.6 & -26.8 & 5.3 & -9.3 & 31.3 & 21.9 \\
\hline
\end{tabular}

${ }^{a}$ Modeled using glycine derivatives. ${ }^{b}$ Located using the $6-31+\mathrm{G}(\mathrm{d})$ basis.

that the qualitative features of the results from the two approaches are similar.

We find (Table 4) that the inclusion of solvation effects does not significantly alter the locations of the variational transition structures. As a consequence, redetermination of the variational transition structures in solution does not significantly change the underlying gas-phase free energy barriers (i.e., $\Delta \Delta G_{\text {gas//solv }}$ is small), except for the abstraction from the $\alpha$-position of the protonated amine and, to a smaller extent, $\beta$-abstraction for both protonated substrates. In addition, solvation corrections $\left(\Delta \Delta G_{\text {solv }}\right)$ act generally in such a way as to increase the free energy barriers associated with hydrogen-atom abstraction from the protonated species. The values of $\Delta \Delta G_{\text {gas } / / \text { solv }}$ and $\Delta \Delta G_{\text {solv }}$ for the neutral systems in Table 4 are much smaller in magnitude and thus do not significantly affect the results. We note that the overall solution-phase barriers $\left(\Delta G_{\text {soln }}{ }^{\ddagger}\right)$ are consistent with the experimental trends of reactivity.

3.3. Hydrogen-Atom Abstractions from Protonated Amino Acids. Abstraction from the $\alpha$-position of protonated amino acids is found to be disfavored both kinetically and thermodynamically. The kinetic deactivation is also apparent at the $\beta$-and, to a smaller extent, $\gamma$-positions, with the barriers becoming smaller, both in the gas phase $\left(\Delta G_{\text {gas }}{ }^{\ddagger}\right)$ and in solution $\left(\Delta G_{\text {soln }}{ }^{\ddagger}\right)$, as one proceeds along the alkyl chain, a result consistent with the experimentally observed regioselectivity (Table 1$).{ }^{13}$ We see that $\Delta \Delta G_{\text {solv }}$ changes monotonically from a negative value at the $\alpha$ position to its largest positive value at the $\varepsilon$-position. In most cases, this is the dominant contribution to the difference between the gas-phase and condensed-phase barriers and serves to compress the range of values of $\Delta G_{\text {soln }}{ }^{\ddagger}$.

An intriguing observation from our calculations is that the gasphase barriers for abstraction from the protonated amine/amino acid are significantly lower at the $\gamma-\varepsilon$-positions, compared with those at the same positions in the neutral structures. These barrier reductions can be attributed to favorable $\mathrm{Cl} \cdot \cdots \mathrm{H}-\mathrm{N}^{+}$interactions that exist between the chlorine atom and charged ammonium moieties in the abstraction transition structures, which are most effective for the conformationally more flexible $\delta$ - and $\varepsilon$-positions, for which cyclic transition structures are observed (Figure 2). As might have been anticipated, solvation leads to a partial washing out of such stabilizing interactions, and thus the barriers in acetic acid solution become larger.

3.4. Comparison with Hydrogen-Atom Abstractions from Species with Protonated Amino Acid Components. With regard to the individual effects of the $\mathrm{NH}_{3}{ }^{+}$and $\mathrm{COOH}$ 
Table 4. Condensed-Phase Reaction Free Energies $\left(\Delta G_{\text {gas }}\right)$, Gas-Phase Variational Free Energy Barriers $\left(\Delta G_{\text {gas }}{ }_{\neq}^{*}\right)$, and Contributions from the Solvent-Induced Geometry Dependence of the Gas-Phase Free Energies $\left(\Delta \Delta G_{\text {gas } / / \text { solv }}{ }^{\mp}\right)$ and Free Energies of Solvation $\left(\Delta \Delta G_{\text {solv }}^{\ddagger}\right)^{a}$ to the Overall Variational Condensed-Phase Free Energy Barriers $\left(\Delta G_{\text {soln }^{\ddagger}}\right)\left(\mathrm{kJ} \mathrm{mol}^{-1}, 298 \mathrm{~K}\right)$

\begin{tabular}{|c|c|c|c|c|c|c|}
\hline Species & & $\Delta G_{\text {soln }}$ & $\Delta G_{\text {gas }}^{\ddagger}$ & $\Delta \Delta G_{\text {gas } / \text { solv }}{ }^{\ddagger a}$ & $\Delta \Delta G_{\text {solv }}^{\ddagger}$ & $\Delta G_{\text {soln }}^{\ddagger}$ \\
\hline & $\alpha^{b}$ & -15.5 & 80.7 & -0.7 & -4.6 & 75.4 \\
\hline $\mathrm{OH}$ & $\beta$ & -30.7 & 30.4 & -6.2 & 11.9 & 36.1 \\
\hline $\mathrm{NH}_{3}{ }^{+}$ & $\gamma$ & -32.9 & 12.4 & 0.0 & 18.5 & 30.9 \\
\hline & $\delta$ & -34.1 & 6.3 & 0.0 & 20.5 & 26.8 \\
\hline & $\varepsilon$ & -34.6 & -3.6 & 0.0 & 23.9 & 20.3 \\
\hline & $\alpha$ & -10.1 & 62.5 & -10.1 & -3.8 & 48.6 \\
\hline $\mathrm{NH}_{3}{ }^{+}$ & $\beta$ & -27.8 & 26.4 & -3.1 & 12.3 & 35.6 \\
\hline & $\gamma$ & -31.6 & 15.2 & 0.0 & 15.1 & 30.3 \\
\hline & $\delta$ & -33.6 & 4.8 & -0.1 & 21.2 & 25.9 \\
\hline & $\varepsilon$ & -35.2 & -2.1 & 0.0 & 23.4 & 21.3 \\
\hline & $\alpha$ & -49.2 & 37.1 & 0.0 & -0.7 & 36.4 \\
\hline $\mathrm{COOH}$ & $\beta$ & -30.8 & 26.8 & 0.0 & 1.2 & 27.9 \\
\hline & $\gamma$ & -32.1 & 22.5 & 0.0 & 0.5 & 23.0 \\
\hline & $\delta$ & -32.8 & 22.9 & 0.0 & -0.1 & 22.8 \\
\hline & $\varepsilon$ & -33.7 & 22.2 & 0.0 & -0.7 & 21.6 \\
\hline & $\alpha$ & -36.4 & 20.2 & 0.0 & -1.2 & 19.0 \\
\hline & $\beta$ & -35.5 & 20.0 & 0.0 & -1.1 & 18.9 \\
\hline & $\gamma$ & -35.7 & 19.9 & 0.0 & -1.1 & 18.8 \\
\hline & $\delta$ & -35.5 & 20.0 & 0.0 & -1.1 & 18.9 \\
\hline & $\varepsilon$ & -36.4 & 20.2 & 0.0 & -1.2 & 19.0 \\
\hline & $\alpha^{\mathrm{b}}$ & -82.0 & 29.6 & -0.6 & 2.4 & 31.3 \\
\hline & $\beta$ & -32.2 & 26.4 & -0.1 & -0.1 & 26.2 \\
\hline NHAC & $\gamma$ & -32.1 & 23.5 & 0.0 & 1.8 & 25.3 \\
\hline & $\delta$ & -33.2 & 22.9 & 0.0 & 0.1 & 23.0 \\
\hline & $\varepsilon$ & -34.6 & 23.3 & 0.0 & -0.3 & 23.0 \\
\hline & $\alpha^{\mathrm{c}}$ & -49.2 & 14.8 & 0.0 & -6.8 & 8.0 \\
\hline NHAC & $\beta$ & -28.0 & 19.6 & 0.0 & 2.0 & 21.6 \\
\hline & $\gamma$ & -33.3 & 21.0 & 0.0 & -0.9 & 20.1 \\
\hline & $\delta$ & -32.9 & 21.0 & 0.0 & -1.7 & 19.2 \\
\hline & $\varepsilon$ & -34.4 & 21.9 & 0.0 & -2.5 & 19.5 \\
\hline
\end{tabular}

${ }^{a}$ Obtained using variational transition structures from the condensed-phase potential energy surface. ${ }^{b}$ Modeled using glycine derivatives. ${ }^{c}$ Located using the $6-31+G(d)$ basis.

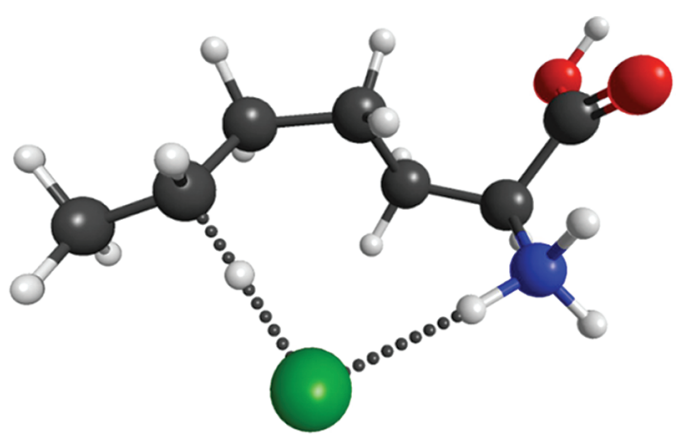

Figure 2. Variational transition structure for abstraction from the $\varepsilon$ position of the protonated amino acid. The calculated bond lengths are $1.245(\mathrm{C} \cdots \mathrm{H}), 1.642(\mathrm{CH} \cdots \mathrm{Cl})$, and $2.203(\mathrm{Cl} \cdots \mathrm{HN}) \AA$.

substituents, the calculated $\Delta G_{\text {soln }}{ }^{\ddagger}$ values (Table 4) indicate that the presence of either of these moieties disfavors abstraction from the $\alpha$-position, and to a smaller extent the $\beta$-position. The calculations are consistent with experimentally observed regioselectivity data available in the case of $\mathrm{COOH} .{ }^{17}$ Comparison of the reaction barriers for hydrogen-atom abstraction by $\mathrm{Cl}^{\bullet}$ from heptane, heptanoic acid, protonated hexylamine, and protonated amino acids indicates that, even at the $\delta$ - and $\varepsilon$-positions, the $\Delta G_{\text {soln }}{ }^{\ddagger}$ barriers for the substituted species still differ by $1-8 \mathrm{~kJ}$ $\mathrm{mol}^{-1}$ from the limiting hydrocarbon barriers of $\sim 19 \mathrm{~kJ} \mathrm{~mol}^{-1}$. It is apparent that the deactivating effect for abstraction at the $\alpha$ and $\beta$-positions is more pronounced in the case of the protonated amine and protonated amino acids than for the carboxylic acid. However, we note that the $\mathrm{COOH}$ group is deactivating for $\alpha$ abstraction, despite the fact that this would lead to the thermodynamically most stable radical product. Thus, it exhibits contrathermodynamic behavior.

These observations can be attributed to inductive or polar field effects, ${ }^{11}$ associated with the expected unfavorable interactions between the electron-withdrawing $\mathrm{COOH}$ and $\mathrm{NH}_{3}{ }^{+}$ groups with the electronegative chlorine in the transition structures. Equally, they are consistent with arguments based on the Pross-Shaik curve-crossing model. ${ }^{8,9}$ In this regard, it has been found that the contribution of the (donor) ${ }^{+}$(acceptor) ${ }^{-}$configuration is important in abstractions by a chlorine atom, leading to the observed polar character. ${ }^{10}$ It is also worth noting that the combined effect of the two substituents in protonated amino acids leads to a larger $\alpha$-abstraction barrier than that found in molecules containing only one of the two functionalities, whereas the side-chain effects with the protonated amino acid are qualitatively similar to those of the protonated amine.

3.5. Hydrogen-Atom Abstractions from N-Acetyl Amino Acids. Our calculations for hydrogen-atom abstractions from 
$N$-acetylated amino acids confirm that abstraction from the $\alpha$ position exhibits remarkable contrathermodynamic behavior, even greater than that seen for the carboxylic acid, in that the product radical is more stable by $\sim 33 \mathrm{~kJ} \mathrm{~mol}^{-1}$ but the barrier is reduced by only $\sim 5 \mathrm{~kJ} \mathrm{~mol}^{-1}$. Thus, while abstraction from the $\alpha$-position of the $N$-acetyl amino acid is associated with a large exergonicity $\left(-82.0 \mathrm{~kJ} \mathrm{~mol}^{-1}\right)$, the $\alpha$-abstraction barrier is the largest for any position along the chain, and larger than the barriers associated with hydrogen-atom abstraction from the $\alpha$-, $\beta$-, and $\gamma$-positions of heptane by approximately $12 \mathrm{~kJ} \mathrm{~mol}^{-1}$. We observe that the variational transition structure for abstraction from $\mathrm{N}$-acetylglycine is particularly early (in acetic acid the $\mathrm{Cl} \cdot \cdots \mathrm{H}$ distance is $\sim 2.07 \AA$ ). This means that the barrier is less influenced by the stabilizing captodative interactions operating within the radical product while being more influenced by the destabilizing inductive effects of the $\mathrm{COOH}$ and acetamido groups. ${ }^{8,10}$

Hydrogen-atom transfer from $N$-hexylacetamide is favored both thermodynamically and kinetically at the $\alpha$-position, but on its own the NHAc substituent has relatively little effect further along an alkyl chain. For $N$-acetyl amino acids, there is a slightly greater deactivation at the $\beta$ - and $\gamma$-positions compared with the $\delta$ - and $\varepsilon$-positions. Combined with the observed significant deactivation at the $\alpha$-position, this is particularly relevant to the resistance of amino acids and peptides and their derivatives toward radical degradation, as the majority of aliphatic hydrogens found in peptides occupy $\alpha-\gamma$-positions, and reactions closer to the backbone generally have more deleterious effects on peptide properties. $^{13}$

\section{CONCLUDING REMARKS}

In summary, we show here that the results of our quantum chemical calculations of hydrogen-atom abstraction barriers are consistent with the experimentally observed regioselectivity of chlorination of protonated and $\mathrm{N}$-acetylated amino acids, in particular the decreased reactivity toward hydrogen-atom abstraction from the backbone $\alpha$-carbon and adjacent side-chain positions. We find no evidence that the observed resistance to hydrogen abstraction by chlorine at the $\alpha$-carbon is associated with the presence of a strong reactant complex, in contrast to a recent suggestion for abstraction by $\mathrm{HO}^{\circ}$. Instead, we find that, for the reactions in the present study, the experimental trends of reactivity can largely be explained by a combination of thermodynamic effects, early transition structures, polar effects (that are particularly important in the early transition structures), variational effects, and solvation.

Among the individual $\mathrm{NH}_{3}{ }^{+}, \mathrm{COOH}$, and $\mathrm{NHAc}$ substituents, we find that the carboxylic acid group is deactivating at the $\alpha$ carbon, despite the fact that abstraction at this position would lead to the most thermodynamically stable radical product. This contrathermodynamic behavior is consistent with the expected unfavorable polar effects destabilizing the transition structure. For $\alpha$-abstraction from the $N$-acetylamino acid, it would seem that the deactivating electrostatic effects of $\mathrm{COOH}$ and $\mathrm{NHAc}$, together with the occurrence of an early transition structure, lead to an even larger contrathermodynamic effect.

In general, the influence of polar and inductive field effects leads to a significant deactivation of backbone and adjacent sidechain positions with respect to hydrogen-atom abstraction by chlorine atom. This supports the hypothesis that the peptides and proteins are peculiarly resistant toward hydrogen-atom abstraction by electrophilic radicals. ${ }^{13}$

\section{ASSOCIATED CONTENT}

S Supporting Information. Full computational details; full set of BHandHLYP/6-31+G(d,p)-optimized transition structures for abstraction from each of the methylene hydrogens from the $\beta-\varepsilon$-positions of the protonated and $N$-acetylated derivatives of $\alpha$-aminoheptanoic acid from which candidate structures were selected for analysis using VTST; comparison of potential energy surfaces for $N$-acetylglycine; optimized geometries (at the BHandH-LYP $/ 6-31+G(d, p)$ level) for structures relevant to the determination of reaction free energies and conventional/variational free-energy barriers; calculated B2K-PLYP/aug'-cc-pV$(\mathrm{T}+\mathrm{d}) \mathrm{Z}$ electronic energies, zero-point vibrational energies, enthalpy and entropy corrections at $298 \mathrm{~K}$, and free energies of solvation in acetic acid for all relevant structures; and complete refs 19,20 , and 26. This material is available free of charge via the Internet at http://pubs.acs.org.

\section{AUTHOR INFORMATION}

\section{Corresponding Author}

radom@chem.usyd.edu.au

\section{ACKNOWLEDGMENT}

We gratefully acknowledge funding (to C.J.E. and L.R.) from the Australian Research Council (ARC), the provision (to RJ.O.) of an Australian Postgraduate Award, and generous grants of computer time (to L.R.) from the National Computing Infrastructure (NCI) National Facility and Intersect Australia Ltd.

\section{REFERENCES}

(1) Garrison, W. M. Chem. Rev. 1987, 87, 381-398.

(2) (a) Davies, M. J.; Dean, R. T. Radical-Mediated Protein Oxidation: From Chemistry to Medicine; Oxford University Press: New York, 1997. (b) Beckman, K. B.; Ames, B. N. Physiol. Rev. 1998, 78, 547-581. (c) Hussain, S. P.; Hofseth, L. J.; Harris, C. C. Nat. Rev. Cancer 2003, 3, 276-285. (d) Mattson, M. P. Nature 2004, 430, 631-639.

(e) Lushchak, V. I. Biochemistry (Moscow) 2007, 72, 809-827. (f) Pitt,

A. R.; Spickett, C. M. Biochem. Soc. Trans. 2008, 36, 1077-1082.

(3) For a review, see: Easton, C. J. Chem. Rev. 1997, 97, 53-82.

(4) Kollonitsch, J.; Doldouras, G. A.; Verdi, V. F. J. Chem. Soc. B 1967, 1093-1095.

(5) Easton, C. J.; Bowman, N. J. J. Chem. Soc., Chem. Commun. 1983, 1193-1194.

(6) Burgess, V. A.; Easton, C. J. Spectrosc. Lett. 1991, 24, 1059-1070.

(7) For a review of experimental work, see: (a) Viehe, H. G.; Janousek, Z.; Merenyi, R.; Stella, L. Acc. Chem. Res. 1985, 18, 148154. For theoretical studies, see for example: (b) Rauk, A.; Yu, D.; Taylor, J.; Shustov, G. V.; Block, D. A.; Armstrong, D. A. Biochemistry 1999, 38, 9089-9096. (c) Croft, A. K.; Easton, C. J.; Radom, L. J. Am. Chem. Soc. 2003, 125, 4119-4124. (d) Wood, G. P. F.; Moran, D.; Jacob, R.; Radom, L. J. Phys. Chem. A 2005, 109, 6318-6325.

(8) Taylor, M. S.; Ivanic, S. A.; Wood, G. P. F.; Easton, C. J.; Bacskay, G. B.; Radom, L. J. Phys. Chem. A 2009, 113, 11817-11832.

(9) Pross, A.; Shaik, S. Acc. Chem. Res. 1983, 16, 363-370.

(10) Pross, A.; Yamataka, H.; Nagase, S. 1991, 4, 135-140.

(11) See for example: Russell, G. A. In Free Radicals; Kochi, J. K., Ed.; Wiley: New York, 1973; Vol. 1, Chapter 7, pp 275-331.

(12) Easton, C. J.; Hay, M. P.; Love, S. G. J. Chem. Soc., Perkin Trans. 1 1988, 265-268.

(13) Watts, Z. I.; Easton, C. J. J. Am. Chem. Soc. 2009, 131, 11323-11325.

(14) $\mathrm{Cl}^{\bullet}$ is used as a representative electrophilic radical. Analogous results have been obtained experimentally for abstractions by $\mathrm{HO}^{\circ}$; see ref 13 and (a) Nukuna, B. N.; Goshe, M. B.; Anderson, V. E. J. Am. Chem. 
Soc. 2001, 123, 1208-1214. (b) Takamoto, K.; Chance, M. R. Annu. Rev. Biophys. Biomol. Struct. 2006, 35, 251-276.

(15) (a) Frey, P. A.; Hegeman, A. D.; Reed, G. H. Chem. Rev. 2006, 106, 3302-3316. (b) Klinman, J. P. Acc. Chem. Res. 2007, 40, 325-333.

(c) Stone, K. L.; Borovik, A. Curr. Opin. Chem. Biol. 2009, 13, 114-118.

(16) For previous theoretical studies of abstractions by chlorine atom in other situations, see for example: (a) Bryukov, M. G.; Slagle, I. R.; Knyazev, V. D. J. Phys. Chem. A 2003, 107, 6565-6573. (b) Tiu, G. C.; Tao, F.-M. Chem. Phys. Lett. 2006, 428, 42-48. (c) Yamanaka, T.; Kawasaki, M.; Hurley, M. D.; Wallington, T. J.; Xiao, L.; Schneider, W. F. J. Phys. Chem. A 2008, 112, 2773-2781. (d) Gruber-Stadler, M.; Mühlhäuser, M.; Sellevåg, S. R.; Nielsen, C. J. J. Phys. Chem. A 2008, $112,9-22$.

(17) Deno, N. C.; Fishbein, R.; Wyckoff, J. C. J. Am. Chem. Soc. 1970, 92, 5274-5275.

(18) Kollonitsch, J.; Doldouras, G. A.; Verdi, V. F. J. Chem. Soc. (B) 1967, 1093-1095.

(19) Frisch, M. J.; Gaussian 03, Revision E.01; Gaussian, Inc.: Wallingford, CT, 2004.

(20) Frisch, M. J.; Gaussian 09, Revision A.02; Gaussian, Inc.: Wallingford, CT, 2009.

(21) The BHandH-LYP transition structure corresponding to hydrogen-atom abstraction from the $\alpha$-position of the $N$-acetylated amine could not be determined using the $6-31+G(d, p)$ basis (that was used for all the other structures) but could be located using the smaller $6-31+G(d)$ basis, and that is therefore what was used as the basis for intrinsic reaction coordinate calculations. It appears from high-level calculations that the abstraction from the $\alpha$-position of the $N$-acetylated amine is barrierless on the vibrationless energy surface.

(22) Tarnopolsky, A.; Karton, A.; Sertchook, R.; Vuzman, D.; Martin, J. M. L. J. Phys. Chem. A 2008, 112, 3-8.

(23) B2K-PLYP/A'VTZ was recently found to produce conventional barriers associated with hydrogen-atom abstractions by $\mathrm{Cl}^{*}$ in good agreement with higher-level URCCSD(T)/aug-cc-pVQZ reference values. See ref 24.

(24) Chan, B.; Radom, L. Theor. Chem. Acc. DOI: 10.1007/s00214011-0967-z.

(25) Merrick, J. P.; Moran, D.; Radom, L. J. Phys. Chem. A 2007, $111,11683-11700$.

(26) Zheng, J.; POLYRATE, version 2010A; University of Minnesota: Minneapolis, MN, 2010.

(27) We found that this mixed basis set performed well, compared with $\mathrm{A}^{\prime} \mathrm{VTZ}$, for studying hydrogen abstraction in a test set of seven systems, including $N$-acetylglycine, $N$-acetylhexylamine, the $\alpha$-position of the heptanoic acid, and a number of alkyl side-chain positions.

(28) Marenich, A. V.; Cramer, C. J.; Truhlar, D. G. J. Phys. Chem. B 2009, 113, 6378-6396.

(29) Similar theoretical results were obtained with carbon tetrachloride, which is often used as the solvent in free-radical chlorination reactions.

(30) Truhlar, D. G.; Isaacson, A. D.; Garrett, B. C. In The Theory of Chemical Reaction Dynamics; Baer, M., Ed.; CRC Press: Boca Raton, FL, 1985; Vol. 4, pp 65-137.

(31) Lu, D.-H.; Truong, T. N.; Melissas, V. S.; Lynch, G. C.; Liu, Y.-P.; Garrett, B. C.; Steckler, R.; Isaacson, A. D.; Rai, S. N.; Hancock, G.; Lauderdale, J. G.; Joseph, T.; Truhlar, D. G. Comput. Phys. Commun. 1992, 71, 235.

(32) Scheiner, S.; Kar, T. J. Am. Chem. Soc. 2010, 132, 16450-16459.

(33) We note that, in Tables 3 and 4, the gas-phase variational free energy barriers $\left(\Delta G_{\mathrm{v}}^{\ddagger}=\Delta G_{\text {gas }}^{\ddagger}\right)$ are less than zero in a small number of cases. This is a result of the formation of moderately strong reactant complexes, with free energies of binding of up to $8.8 \mathrm{~kJ} \mathrm{~mol}^{-1}$. Reactant complexes are also found for several reactions for which the gas-phase barriers are positive, but in these cases the interactions are far weaker. In the condensed phase, the effects of solvation effectively quench this electrostatic interaction, and the free energy barrier $\left(\Delta G_{\text {soln }}{ }^{*}\right)$ becomes positive.

(34) Malick, D. K.; Petersson, G. A.; Montgomery, J. A. J. Chem. Phys. 1998, 108, 5704. 